\title{
EXPERIMENTAL VERIFICATION OF GAP METRIC AS A TOOL FOR MODEL SELECTION IN MULTI-LINEAR MODEL-BASED CONTROL
}

\author{
Omar Galán ${ }^{1}$, Jose A. Romagnoli ${ }^{2}$, Ahmet Palazoğlu ${ }^{3}$, Yaman Arkun ${ }^{4}$ \\ ${ }^{1}$ ABB Australia Limited Pty, VPP9 Project, 436 Gadara Road, Tumut NSW 2720 AUSTRALIA, ${ }^{2}$ Dept. of Chemical \\ Engineering, The University of Sydney, Sydney, NSW, 2006, AUSTRALIA, ${ }^{3}$ Dept. of Chemical Engineering and \\ Materials Science, University of California, Davis, CA 95616 USA, ${ }^{4}$ College of Engineering, Koç University, \\ Rumelifeneri, Sariyer, İstanbul, 80910 TURKEY
}

\begin{abstract}
A nonlinear system can be modeled using a set of linear models that cover the range of operation. A model-based control strategy then can be employed that uses the local models in a cooperative manner to control the nonlinear system. The decision of how many models are sufficient for effective control can be tackled by the use of the gap metric that quantifies the distance between two linear operators. A pH control experiment is used to demonstrate the effectiveness of gap metric as a tool for model selection. Copyright $\odot 2003$ IFAC

Keywords: model-based control, nonlinear systems
\end{abstract}

\section{INTRODUCTION}

Classical linear design tools have matured to a point where one can incorporate robustness and performance requirements in a natural fashion. However for nonlinear processes strictly linear designs may not provide satisfactory performance unless they are suitably modified. One approach which tries to keep the features of linear design and at the same time account for nonlinearities is the multi-model approach for controller design ( $\mathrm{Yu}$ et al., 1992; Murray-Smith and Johansen, 1997; Özkan et al., 2003). The key concept is to represent the nonlinear system as a combination of linear systems where classical control design techniques can be applied. The controller design based on the multimodel approach requires either simultaneous plants stabilization using a single controller, subject to performance and stability constrains (Schöming et al, 1995; Galán et al, 2000), or interpolation using model validity functions, where local controllers are selected as a function of the current state of the process (Foss et al, 1995; Banerjee et al, 1997). However, in all these approaches the question of how many and which models are required remains largely unanswered. Although it is common to use a large number of local models to improve the piece-wise linear approximation of the nonlinear system (Narendra et al, 1995), the optimization problem to solve the design problem becomes formidable when the number of local models is large.

We shall formulate the multi-model control problem by assuming a set of local plants and controllers that stabilize these plants and by asking the question, "Is there a reduced set of controllers, which are based on models that are "close' in some sense?"

To determine when two systems are close to one other is a nontrivial task, and furthermore, what is meant by "close" is not entirely obvious. Since systems can be visualized as input-output operators, a natural distance concept would be the induced operator norm. Yet, the norm cannot be generalized as a distance measure (Vidyasagar, 1985). The aim of this paper is to discuss the application of a distance measure between systems, the so-called Gap Metric, to select a reduced set of models that contain non-redundant process information for robust stabilization of feedback systems based on multimodel controller design

\section{GAP METRIC}

The concept of the gap between the graphs of two linear systems goes back to Hausdorf (1935). Later the gap and other metrics were used to study how close different operators are (e.g. Newburgh (1951), 
Berkson (1963)). In Zames and El-Sakkary (1980) the gap metric was used to establish a topology to quantify the tolerable uncertainties, which preserve closed loop stability. El-Sakkary (1985) shows that the gap metric is better suited to measure the distance between two linear systems than a metric based on norms. For more details, the reader is referred to these references and Galan et al. (2002).

Let $P$ be a finite dimensional linear system. Its transfer function will be denoted by $\hat{P}(s)$. The transfer function $\hat{P}(s)$ can be expressed by a normalized right coprime factorization:

$$
\hat{P}(s)=\hat{N}(s) \hat{D}^{-1}(s)
$$

where $\hat{N}$ and $\hat{D}$ belong to the subspace of real rational functions in $H_{\infty} ; \hat{D}$ has a proper inverse, and

$$
\hat{D}^{*} \hat{D}+\hat{N}^{*} \hat{N}=I
$$

where $\hat{D}^{*}(s)=\hat{D}(-s)^{T}$. These factorizations can be computed using existing techniques (Vidyasagar, 1988).

The graph of the operator $P$ is the subspace of $\mathrm{H}_{2} \times \mathrm{H}_{2}$ (Hardy space of functions) that consists of all pairs $(u, y)$ such that $y=P u$. This is expressed as

$$
\operatorname{graph}(P)=\left(\begin{array}{c}
D \\
N
\end{array}\right) H_{2} \equiv G H_{2}
$$

where the operator $\left(\begin{array}{c}D \\ N\end{array}\right)$ is denoted by $G$.

Let $\Pi_{G}$ be the orthogonal projection operator which maps any element $\{x, y\}$ in $H \times H$ to $\{u, P u\}$ and is given by:

$$
\Pi_{G}=G G^{*}
$$

The calculation of the gap metric begins with two finite dimensional linear systems with the same number of inputs and outputs whose normalized coprime factorizations are given by:

$$
\hat{P}_{i}(s)=\hat{N}_{i}(s) \hat{D}_{i}^{-1}(s)
$$

and their respective "graph" operators $G_{i}$ are defined as above for $i=1$ and 2 .

It can be shown that the directed gap can be computed using the projection operators or the coprime factorizations (Georgiou, 1988):

$$
\begin{aligned}
& \bar{\delta}\left(P_{1}, P_{2}\right)=\left\|\left(I-\Pi_{G_{2}}\right) \Pi_{G_{1}}\right\| \\
& =\inf _{Q \in H_{x}}\left\|\left(\begin{array}{c}
D_{1} \\
N_{1}
\end{array}\right)-\left(\begin{array}{c}
D_{2} \\
N_{2}
\end{array}\right) Q\right\|_{\infty}
\end{aligned}
$$

Definition 2 (Georgiou, 1988): The gap between two systems $P_{1}$ and $P_{2}$ is given by:

$$
\delta\left(P_{1}, P_{2}\right)=\max \left\{\vec{\delta}\left(P_{1}, P_{2}\right), \vec{\delta}\left(P_{2}, P_{1}\right)\right\}
$$

and using (6) one gets

$$
\max \left\{\begin{array}{c}
\inf _{Q \in H_{x}}\left\|\left(\begin{array}{c}
D_{1} \\
N_{1}
\end{array}\right)-\left(\begin{array}{c}
D_{2} \\
N_{2}
\end{array}\right) Q\right\|_{\infty}, \\
\inf _{Q \in H_{\infty}}\left\|\left(\begin{array}{c}
D_{2} \\
N_{2}
\end{array}\right)-\left(\begin{array}{c}
D_{1} \\
N_{1}
\end{array}\right) Q\right\|
\end{array}\right\}
$$

Properties of the gap:

1. The gap defines a metric on the space of (possibly unstable) linear systems.

2. $0 \leq \delta\left(P_{1}, P_{2}\right) \leq 1$

The metric defines a notion of distance in the space of (possibly unstable) linear systems, which do not assume that plants have the same number of poles in the RHP.

The computation of the gap involves solving twoblock $H_{x}$ problems (7). In our examples, we used MATLAB $\mu$-Synthesis Toolbox to compute the gap.

If the gap metric is close to zero, it indicates that the distance between two systems is 'close'. If, on the other hand, the gap is closer to 1 ; then, the two systems' dynamic behaviors are 'apart'. In the following experimental study, we use the gap metric to distinguish between models in a given set. We use the gap analysis to select appropriate linear models to control a $\mathrm{pH}$ neutralization system using a multilinear controller.

\section{EXPERIMENTAL STUDY}

The model of the process for $\mathrm{pH}$ neutralization is taken from the paper by Galán et al. (2000). Based on that model, first-order transfer function models are derived for five distinct operating regions in the steady-state map (Fig. 1).

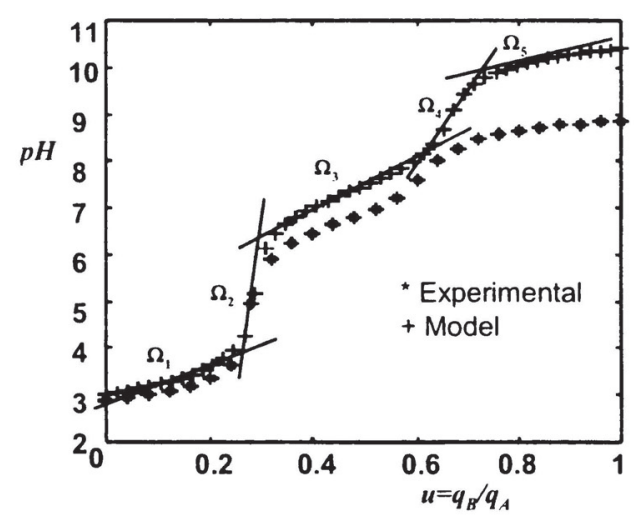

Fig. 1. The steady-state map of the process and the model prediction, indicating five regions for local linear models.

Table 2 shows the gap metric between the pairs of five linear models representing the operating range for the nonlinear system (Fig. 1). The numbers suggest that model subsets $\Omega_{H S}=\left\{\Omega_{2}, \Omega_{4}\right\}$ and $\Omega_{L S}=\left\{\Omega_{1}, \Omega_{3}, \Omega_{5}\right\}$ are closer to being "different" while the members in each subset are closer to being "similar." Model 2 is considered different than 
models 1,3 and 5 as the gap metric is around 1, but close to model 4 . This similarity between models 2 and 4 can be explained physically by the fact that those models represent high sensitivity regions " $\Omega_{H S}$ ", that is, regions with a steep slope (Fig. 1). It contrasts with models 1,3 and 5 , which represent the low sensitivity regions " $\Omega_{L S}$ ".

Table 2. Distance between Linear Models Using Gap

\begin{tabular}{cccccc}
\multicolumn{6}{c}{ Metric. } \\
\hline$\delta$ & 1 & 2 & 3 & 4 & 5 \\
\hline 1 & 0.0000 & 0.9331 & 0.2561 & 0.8050 & 0.2619 \\
2 & 0.9331 & 0.0000 & 0.8885 & 0.5137 & 0.9515 \\
3 & 0.2561 & 0.8885 & 0.0000 & 0.6892 & 0.4183 \\
4 & 0.8050 & 0.5137 & 0.6892 & 0.0000 & 0.8567 \\
5 & 0.2619 & 0.9515 & 0.4183 & 0.8567 & 0.0000 \\
\hline
\end{tabular}

\subsection{Controller Design}

Consider a set of $N$ local linear models that, in combination, describe the behavior of a nonlinear system in a pre-defined operating range. The key issue is how one implements the multi-model control scheme with a set of local controllers that are derived using the local models. For this example, the controllers for different local operating regions are combined to form a complete control system, using membership functions to create a transition region in the measured variable " $y$ " (de Silva and MacFarlane, 1989).

$$
\hat{\phi}_{p}(y)=\frac{\phi_{p}}{\sum_{p=1}^{n} \phi_{p}}
$$

The subscript $p$ represents the $p^{\text {th }}$ member of a set of $N$ controllers. Given the output variable $y$, the membership function returns a number between zero and one indicating the level of contribution of the local controller at that value of the output. We define the distribution function for a local controller,

$$
\phi_{p}(y)=\exp \left[-\frac{1}{2}\left(\frac{y-\bar{y}_{p}}{\sigma_{p}}\right)^{2}\right]
$$

where $\bar{y}_{p}$ and $\sigma_{p}$ are the mean and the standard deviation related to the model " $p$ ", respectively. The desired contribution of combined controllers on the control signal can be represented as a function of the membership functions:

$$
u(t)=\sum_{p=1}^{N} \hat{\phi}_{p} u_{p}(t)
$$

The block diagram for the local controllers is given in Figure 2. Given a SISO plant $\hat{g}_{p}(s)$, a controller $\hat{k}_{p}(s)$ is designed such that the basic requirements of stability, performance and robustness are satisfied
(Doyle et al., 1992). This can be done by finding a robust controller that minimizes the mixed-sensitivity criterion (Skogestad and Postletwaite, 1996),

$$
\begin{aligned}
& J=\sup _{\omega}\left|\begin{array}{c}
\hat{w}_{e}(s) \hat{S}_{p}(s) \\
\hat{w}_{u}(s) \hat{k}_{p}(s) \hat{S}_{p}(s) \\
\hat{w}_{y}(s) \hat{T}_{p}(s)
\end{array}\right| \\
& =\left\|\begin{array}{c}
\hat{w}_{e}(s) \hat{S}_{p}(s) \\
\hat{w}_{u}(s) \hat{k}_{p}(s) \hat{S}_{p}(s) \\
\hat{w}_{y}(s) \hat{T}_{p}(s)
\end{array}\right\|_{\infty}
\end{aligned}
$$

Note that $\hat{S}_{p}(s)$, and $\hat{T}_{p}(s)$ are the local sensitivity and complementary sensitivity functions respectively. Accordingly, $\hat{w}_{\mathcal{e}}, \hat{w}_{u}$ and $\hat{w}_{y}$ are the corresponding weight (penalty) functions chosen to shape the closed-loop performance and robustness behavior

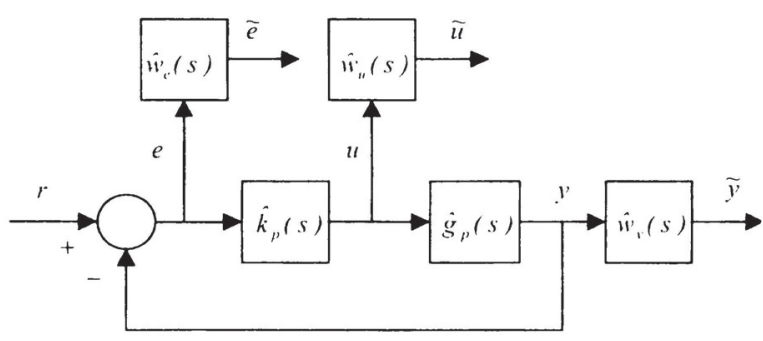

Fig. 2. Block diagram for the local closed-loop system.

\subsection{Experimental verification}

To study the closed-loop performance of these controllers based on a subset of models, real-time experiments are performed. An acid stream $(\mathrm{HCl}$ solution) and an alkaline stream $\left(\mathrm{NaOH}\right.$ and $\mathrm{NaHCO}_{3}$ solution) are fed to a 2.5-liter constant volume, wellmixed tank, where the $\mathrm{pH}$ is measured through a sensor located directly in the tank. The control objective is to drive the system to different $\mathrm{pH}$ conditions (tracking control) by manipulating the alkaline stream flowrate.

Figure 3 shows the tracking performance when all five models are included. The response is generally acceptable, but the aggressive behavior around $\mathrm{pH}=5$ (region 2) is noted. We can compare this performance with the case where only three models (from $\Omega_{L S}$ ) are included. Figure 4 shows that there is clearly a loss of performance, especially around region 2. This validates what we have seen before in the gap metric analysis, as these models are not sufficiently descriptive of the whole operating region.

When only two models 1 and 2 or 2 and 3 are used in combination (one model from $\Omega_{L S}$ and one model from $\Omega_{H S}$ ), the results are given in Figs. 5 and 6 , respectively 

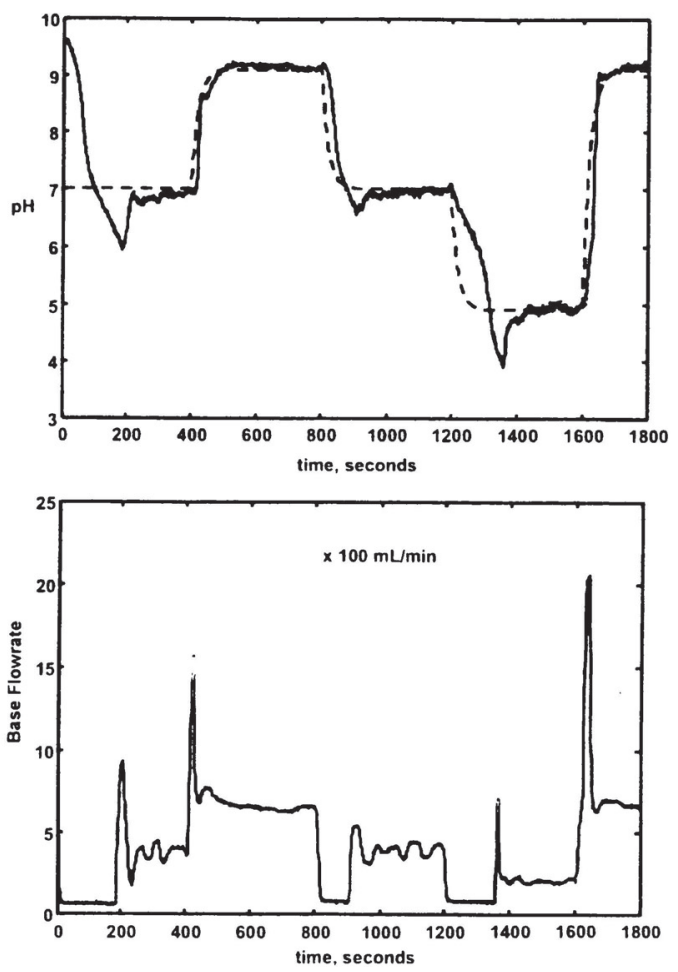

Fig. 3. Tracking performance when all five models used.
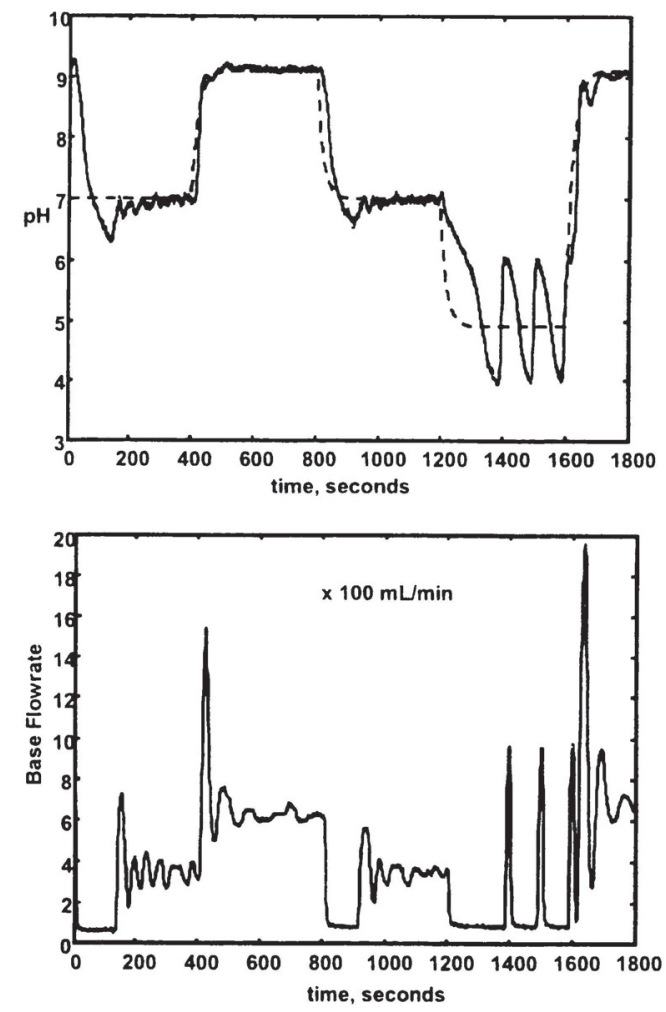

Fig. 4. Tracking performance when only models from the set $\Omega_{L S}$ are used.

The responses are quite similar to the one obtained by using all five models as models in region 2 and 4 sufficiently explain the dynamics within the operating region. In fact, the control action appears to be much smoother when only two models are considered. The results also indicate that the first combination $(1,2)$ exhibits some degradation in performance while the second $(2,3)$ offers a more satisfactory tracking behavior (especially in the control action).
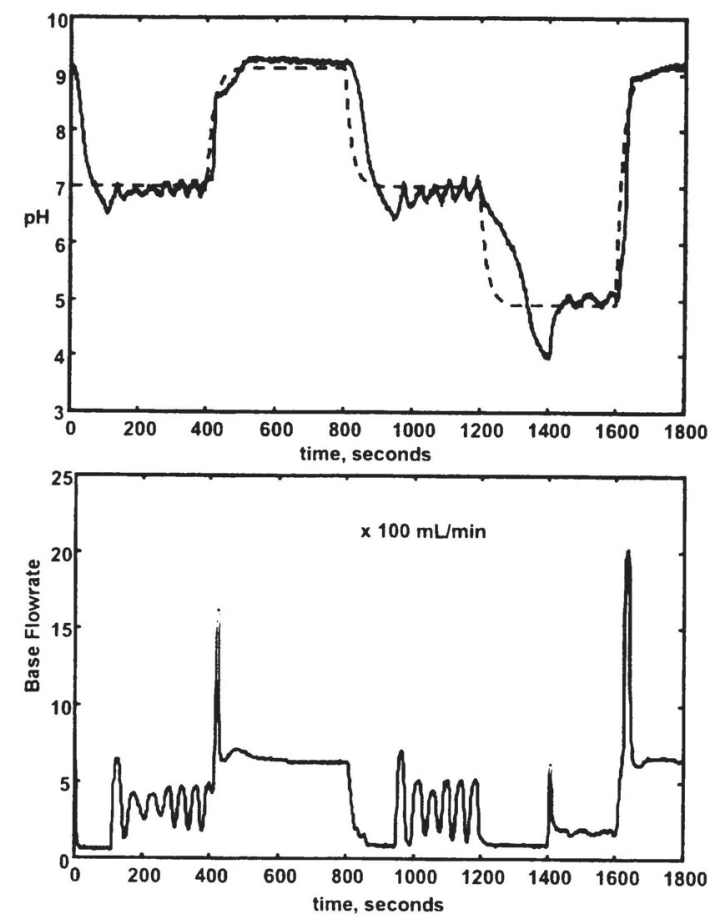

Fig. 5. Tracking performance when two models used, one from each sensitivity region.
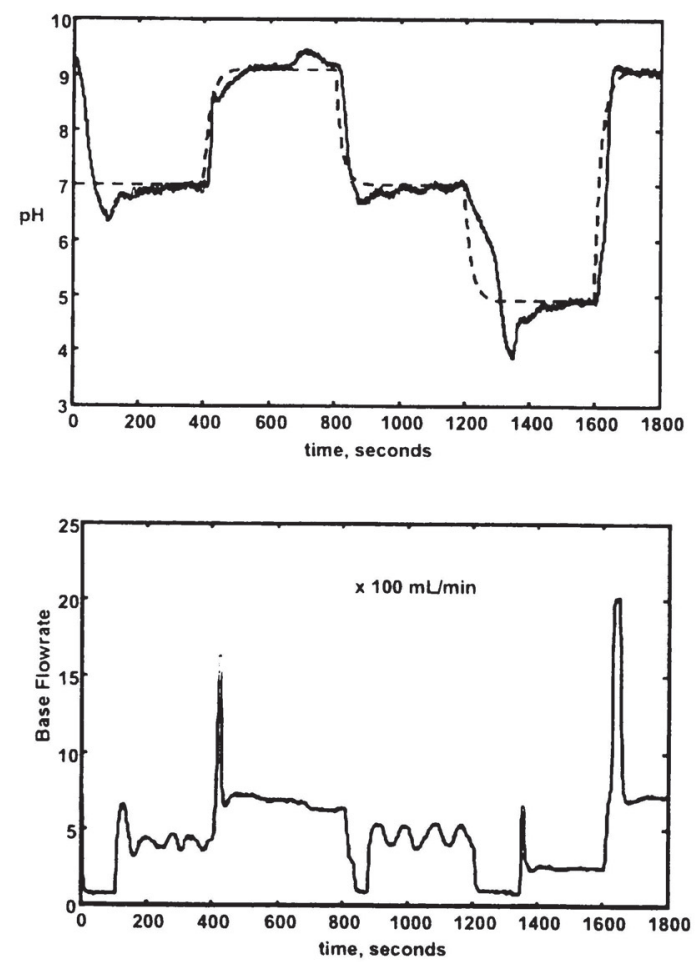

Fig. 6. Tracking performance when two models used, one from each sensitivity region. 


\section{CONCLUSIONS}

The results of the case study using gap metric, as a measure of the distance between two linear plants, suggests the potential for a rigorous measure to evaluate the number of models in multi-linear modelbased control. For the case of $\mathrm{pH}$ neutralization where one intuitively would use five models to cover the $\mathrm{pH}$ range between 3 and 10 , we have shown that two models may be sufficient to guarantee satisfactory closed-loop performance.

Acknowledgements: A grant from the Australian Research Council (ARC-A89906571) is gratefully acknowledged.

\section{REFERENCES}

Banerjee, A, Arkun, Y., Ogunnaike, B., Pearson, R., (1997). "Estimation of Nonlinear Systems Using Linear Multiple Models," AIChE J., 43, 12041226.

Berkson, E., (1963). "Some Metrics on the Subspaces of a Banach Space," Pacific J. Math, 13, 7-22.

de Silva, C.W., and MacFarlane, A.G.J., (1989). Knowledge-Based Control with Application to Robots, Springer-Verlag, NY.

Doyle, J.C., Francis, B.A. and Tannenbaum, A.R., (1992). Feedback Control Theory, Macmillan, New York, NY.

El-Sakkary, A., 1985, "The Gap Metric: Robustness of Stabilization of Feedback Systems," IEEE Trans. on Automatic Control, AC-30, 240-247.

Foss, B.A., Johansen, T.A. and Sorensen, A.V., 1995, "Nonlinear Predictive Control Using Local Models - Applied to a Batch Fermentation Process," Control Eng. Practice, 3, 389-396.

Galán, O., Palazoglu, A. and Romagnoli, J. A., 2000, "Robust $H_{\infty}$ Control for Nonlinear Plants Based on Multi Linear Models - An Application to a Bench Scale pH Neutralization Reactor," Chem. Eng. Sci., 55, 4435-4450.

Galán, O., Romagnoli, J. A., Palazoglu, A. and Arkun, Y. (2002). "The Gap Metric Concept and Implications for Multi-Linear Model-Based Controller Design," Ind. \& Engg Chem., Research, submitted.

Georgiou, T.T., 1988, "On the Computation of the Gap Metric," Systems \& Control Letters, 11, 253-257.

Hausdorff, F.,(1935). Mengenlehre, Berlin: Leipzig.

Murray-Smith, R., and Johansen, T.A. (eds.), 1997, Multiple Model Approaches to Modeling and Control, Taylor \& Francis, London, England.

Narendra, K.S., Balakrishnan, J. and Ciliz, M.K., 1995, "Adaptation and Learning Using Multiple Models, Switching and Tuning," IEEE Control Syst. Magazine, 15, 37-51.

Newburgh, J. D., 1951, "A Topology for Closed Operators," Ann. Math., 53, 250-255.

Özkan, L., Kothare, M.V., and Georgakis, C., 2003, "Control of a Solution Copolymerization
Reactor Using Multi-Model Predictive Control," Chem. Eng. Sci., in press.

Schöming, E., Sznaier, M. and Ly, U., 1995, "Mixed $\mathrm{H}_{2} / \mathrm{H}_{\infty}$ : Control of Multimodel Plants," $J$. Guidance, Control and Dynamics, 18, 525-531.

Skogestad, S. and Postlethwaite, I., 1996, Multivariable Feedback Control, Wiley, New York, NY

Vidyasagar, M., 1985, Control System Synthesis: A Factorization Approach, MIT Press, Cambridge, $\mathrm{MA}$

Vidyasagar, M., 1988, "Normalized Coprime Factorizations for Nonstrictly Proper Systems," IEEE Trans. Automat. Control, 33, 300-301.

Yu, C., Roy, R.J., Kaufman, H., and Bequette, B.W., 1992, "Multiple-Model Adaptive Predictive Control of Mean Arterial Pressure and Cardiac Output," IEEE Trans. Biomed.Eng., 39, 765-778.

Zames, G. and El-Sakkary, A.K., 1980, "Unstable Systems and Feedback: The Gap Metric," Proceedings of the Allerton Conf., 380-385. 